\title{
Efeito de Hypericum caprifoliatum Cham. \& Schltdl. (Guttiferae) sobre contrações em íleo isolado de cobaio induzidas por diferentes agonistas
}

\author{
Alice F. Viana ${ }^{1,2}$, Ana P. M. Heckler', Gilsane Lino von Poser ${ }^{1,2}$, Augusto Langeloh ${ }^{3}$, \\ Stela M. K. Rates ${ }^{1,2 *}$ \\ ${ }^{1}$ Programa de Pós-Graduação em Ciências Farmacêuticas, Faculdade de Farmácia, Universidade Federal do \\ Rio grande do Sul, Av. Ipiranga 2752, 90610-000, Porto Alegre, RS, Brasil, \\ ${ }^{2}$ Laboratório de Psicofarmacologia Experimental, Faculdade de Farmácia, Universidade Federal do Rio grande \\ do Sul, Av. Ipiranga 2752, 90610-000, Porto Alegre, RS, Brasil, \\ ${ }^{3}$ Departamento de Farmacologia, Instituto de Ciências Básicas da Saúde, Universidade Federal do Rio Grande \\ do Sul, Av. Sarmento Leite 500, 90040-060, Porto Alegre, RS, Brasil
}

\begin{abstract}
RESUMO: Na última década, o gênero Hypericum ganhou repercussão mundial devido à utilização de Hypericum perforatum para obtenção de medicamentos antidepressivos. Por esta razão, a maioria dos estudos com outras espécies do gênero centra-se nesta atividade. Porém, um dos usos populares de espécies de Hypericum nativas do sul do Brasil é no tratamento de problemas gastrintestinais, inclusive como antiespasmódico. Neste trabalho, foi avaliado o efeito de uma das espécies de Hypericum nativas do Rio Grande do Sul, H. caprifoliatum, sobre as contrações induzidas por agonistas em íleo isolado de cobaio. Foi investigado o efeito de um extrato ciclo-hexano purificado (isento de clorofila e ceras), nas concentrações de 1, 3, 10 e $30 \mathrm{mg} / \mathrm{mL}$, sobre curvas cumulativas de acetilcolina, histamina, potássio e serotonina $\left(10^{-7} \mathrm{a}\right.$ $\left.10^{-4} \mathrm{M}\right)$. Na concentração de $30 \mathrm{mg} / \mathrm{mL}$ o extrato inibiu totalmente as contrações induzidas por todos os agonistas. Na concentração de $10 \mathrm{mg} / \mathrm{mL}$, o extrato apresentou efeito antagonista nãocompetitivo de serotonina, reduzindo a contração máxima induzida por serotonina em cerca de $50 \%$. A resposta contrátil aos outros mediadores não foi alterada. Estes resultados indicam que espécies de Hypericum do sul do Brasil podem ser uma perspectiva interessante na busca de moléculas com atividade sobre a motilidade gastrintestinal.
\end{abstract}

Unitermos: Hypericum caprifoliatum, íleo de cobaio, serotonina, antiespasmódico.

\begin{abstract}
Effect of Hypericum caprifoliatum Cham \& Schltdl (Guttiferae) on isolated guinea pig ileum serotonin response”. In the last decade the genus Hypericum has achieved worldwide recognition due to the therapeutic value of $H$. perforatum as an antidepressant drug. Consequently this activity is the most investigated one. However, species native to Brazil have other folk uses such as for the treatment of digestive disorders, including cramps. In this study we evaluated the effect of a purified cyclohexane extract (chlorophyll and waxes free) $(1,3,10$ and $30 \mathrm{mg} / \mathrm{mL}$ ) of $H$. caprifoliatum, a specie native to South Brazil, on isolated guinea pig ileum contractions induced by different mediators: serotonin, histamine, acetylcholine and potassium chloride $\left(10^{-7}-10^{-4} \mathrm{M}\right)$. At $30 \mathrm{mg} / \mathrm{mL}$ all contractile responses were abolished. At $10 \mathrm{mg} / \mathrm{mL}$ only serotonin responses were altered: the extract reduced the maximal effect in 50\%, which represents a non-competitive antagonism. At 1 and $3 \mathrm{mg} / \mathrm{mL}$ the extract was unable to modify all mediators response. These results point to native species of Hypericum as an interesting perspective in searching new molecules active on gastrointestinal motility.
\end{abstract}

Keywords: Hypericum caprifoliatum, guinea pig ileum, serotonin, spasmolytic.

\section{INTRODUÇÃO}

O gênero Hypericum ganhou repercussão mundial devido à utilização de Hypericum perforatum para obtenção de medicamentos antidepressivos. A espécie Hypericum perforatum é uma planta muito utilizada na cultura anglo-saxã, onde é denominada popularmente como erva-de-são joão (Saint John wort, Johanniskraut). Esta espécie vem sendo utilizada desde a Grécia antiga como cicatrizante, antiinflamatório, anti-séptico e no alívio de sintomas de ansiedade e depressão. Estes usos foram descritos por Dioscorides, I d.C.; Galeno, II d.C.; Plínio, em livro XXIV de Historiarum mundi; e Paracelso, na Idade Média (Duke, 
1985). Ainda hoje, extratos oleosos são utilizados na medicina popular para o tratamento de ferimentos, herpes, úlceras externas, gota, reumatismo, mialgia e queimaduras de primeiro grau (Duke, 1985; Malamas; Marselos, 1992; Blumenthal, 1998; Reichling et al., 2001); infusões das flores são usadas como adstringente, diurético, antidepressivo e ansiolítico (Reynolds, 1993; Blumenthal, 1998). No contexto da medicina científica, os extratos de Hypericum perforatum são os produtos de origem vegetal com maior número de estudos clínicos no mundo e representam uma alternativa aos antidepressivos sintéticos (Ernst, 2000; Bilia et al., 2002; Linde; Knuppel, 2005; Kasper et al., 2006).

No Brasil, existem registros de uso na medicina popular das seguintes espécies: $H$. teretiusculum como excitante, aromática e emenagoga; $H$. laxiusculum como adstringente, aromática, vulnerária, antiespasmódica e para tratamento de picada de cobra; $H$. guianense, como purgativo e para infecções da pele; $H$. connatum, como tônica, adstringente e no tratamento de anginas, aftas e estomatite; H. latifolium (Vismia latifolium) como tônica e febrífuga (Corrêa, 1984). O maior número de estudos científicos publicados com as espécies brasileiras é relativo à atividade antimicrobiana in vitro (Rocha et al., 1994, 1995; Schimitt et al., 2001; Dall'Agnol et al., 2003; Fenner et al., 2005); atividade alelopática (Fritz et al., 2007) e desenvolvimento embionário em ratas (Nepomuceno et al., 2005). Também foram verificadas atividades antiproliferativa (Ferraz et al., 2005), inibidora de monoaminoxidase (IMAO) (Rocha et al., 1994; Gnerre et al., 2001), antidepressiva (Daudt et al., 2000; Gnerre et al., 2001; Viana et al., 2005) e analgésica (Viana et al., 2003).

Viana e col. (2003) verificaram que o extrato ciclo-hexano de $H$. caprifoliatum apresenta efeito antinociceptivo nos testes da placa aquecida e de contrações abdominais induzidas por ácido acético 0,8\%. Este último teste avalia a dor ocasionada pela ativação de quimio-receptores viscerais (Bjorkman, 1995), porém não é específico, respondendo a diferentes classes de agentes analgésicos e também a antagonistas adrenérgicos, anti-histamínicos $\mathrm{H}_{1}$, miorelaxantes, antipsicóticos (Le Bars et al., 2001). Deste modo, o presente estudo foi realizado com o objetivo de investigar quais mediadores periféricos estão envolvidos no efeito inibidor das contrações abdominais do extrato ciclo-hexano de $H$. caprifoliatum, avaliando seu efeito sobre contrações em íleo isolado de cobaio induzidas por diferentes agonistas.

\section{MATERIAL E MÉTODOS}

\section{Animais}

Foram utilizados cobaios machos, com peso entre 300 - 400 g, obtidos do Laboratório do Ministério da Agricultura, em Porto Alegre. Antes dos experimentos, os animais foram adaptados por 72 h no biotério da Faculdade de Farmácia - UFRGS, mantidos um a um em caixas plásticas com livre acesso a água e alimento, em ciclo de claro/escuro de 12 h (07:00 - 19:00 h), em ambiente com temperatura controlada $\left(23 \pm 2{ }^{\circ} \mathrm{C}\right)$ e umidade monitorada. Os animais foram levados para a sala de experimentação uma hora antes do início dos testes.

Os protocolos experimentais foram aprovados pelo Comitê Nacional de Ética em Pesquisa, representado em Porto Alegre pela Comissão Científica e Comissão de Pesquisa e Ética em Saúde do Hospital de Clínicas de Porto Alegre (Projeto 01-588).

\section{Material vegetal}

As partes aéreas de Hypericum caprifoliatum Cham. \& Schltdl. foram coletadas em Viamão / RS, em dezembro de 2002. A exsicata do material vegetal preparada para identificação foi registrada no herbário ICN (Herbário do Departamento de Botânica - Instituto de Biociências - UFRGS) sob os números Bordignon 1400 (H. caprifoliatum). O material, imediatamente após a coleta, foi selecionado, seco em ambiente arejado, ao abrigo da luz direta e rasurado manualmente.

\section{Drogas e solventes}

Acetilcolina, cloreto de potássio, histamina e serotonina, de procedência Sigma (St. Louis/ US); ciclo-hexano, dimetilsulfóxido e polissorbato 80, de procedência Merck (Darmstadt/ DE).

\section{Extrato}

O material vegetal foi submetido à extração por maceração utilizando-se ciclo-hexano. Foram realizadas três extrações de 12 h empregando a proporção droga/ solvente de 1:10 (p/V), o solvente foi eliminado com evaporador rotatório $\left( \pm 45^{\circ} \mathrm{C}\right)$, sob pressão reduzida. $\mathrm{O}$ extrato ciclo-hexano seco foi ressuspendido em acetona. A adição deste solvente torna as ceras insolúveis, ocorrendo formação de precipitado, o qual foi removido por filtração em papel. Após evaporação sob pressão reduzida $\left( \pm 45{ }^{\circ} \mathrm{C}\right)$, o resíduo seco do filtrado foi denominado HCP, com rendimento de c.a. $3,5 \%$, em relação à droga seca. HCP apresenta como composto majoritário (8\%) um derivado de floroglucinol denominado HC1, caracterizado conforme descrito por Daudt et al. (2000) e Viana et al. (2005).

\section{Ensaio do íleo isolado de cobaio}

Foram utilizadas as condições adaptadas a partir de Chatterjee et al. (1998). Os cobaios foram sacrificados por contusão craniana, exsangüinados, e a porção terminal do íleo foi removida, sendo utilizados 
fragmentos de $2 \mathrm{~cm}$. A preparação foi montada em banho de órgão isolado contendo solução de Tyrode (g/L): $\mathrm{NaCl} 8, \mathrm{KCl} 0,2 ; \mathrm{CaCl}_{2} 0,265 ; \mathrm{MgSO}_{4} 0,2$; $\mathrm{NaHCO}_{3} 1,0 ; \mathrm{NaH}_{2} \mathrm{PO}_{4}$ 0,1; glicose 1,0 - pH 7,4 em temperatura de $37^{\circ} \mathrm{C}$, com aeração constante. Após 30 min de estabilização do órgão às condições do banho, foi registrado o grau de contração basal do órgão (tônus) submetido a $1 \mathrm{~g}$ de carga, com auxílio de alavanca isotônica, ampliação de 6 vezes. Posteriormente, foram realizadas, separadamente, em diferentes preparações, curvas cumulativas $\left(10^{-7}\right.$ a $\left.10^{-4} \mathrm{M}\right)$ com acetilcolina (Ach), histamina, cloreto de potássio $(\mathrm{KCl})$ e serotonina (5-HT).

O HCP foi ressuspenso em solução salina com auxílio de polissorbato 80, numa concentração final no banho não superior a $0,1 \%$. A suspensão foi adicionada ao banho nas concentrações de 1, 3, 10 e $30 \mathrm{mg} / \mathrm{mL}$ e após 10 minutos foi realizada uma nova curva com cada agente espasmogênico.

\section{Análise dos dados}

A afinidade relativa dos agonistas $\left(\mathrm{pD}_{2}\right)$ foi calculada por regressão linear utilizando-se o programa
Curve Expert 1.3 (copyright: Daniel Hyams) e a concentração capaz de produzir $50 \%$ do efeito $\left(\mathrm{CE}_{50}\right)$ foi calculada pela equação:

$$
\mathrm{pD}_{2}=-\log \mathrm{CE}_{50}
$$

Quando se trabalha com extratos de plantas não é possível determinar os valores de $\mathrm{pA}_{2}$ (afinidade do antagonista competitivo) e $\mathrm{pD}_{2}{ }_{2}$ (afinidade do antagonista não competitivo) matematicamente, uma vez que não há como determinar a concentração molar do antagonista. Nesta situação, calcula-se a concentração de extrato necessária para deslocar a curva concentração-efeito para a direita num fator de 2 , representada por $\mathrm{CD}_{2} \mathrm{e} / \mathrm{ou}$ a concentração inibitória do efeito máximo do agonista em $50 \%$, representada por CIEM $_{50}$ (Amaral, 2000). A afinidade do antagonista $\left(\mathrm{pD}_{2}^{\prime}\right)$ foi calculada através da regressão linear do gráfico de Schild modificado (Áries; van Rossum, 1957), que relaciona o logaritmo de (ER1) com o logaritmo da concentração do extrato, onde ER é a relação dos efeitos máximos do controle (ausência de antagonista) e das curvas de agonistas na presença de antagonista. Através do antilogaritmo de $\mathrm{pD}_{2}^{\prime}$ calculou-se a CIEM50 (concentração inibidora do efeito máximo do agonista em 50 \%). O possível antagonismo

Tabela 1. Variáveis farmacológicas de 5-HT, acetilcolina, histamina e $\mathrm{KCl}$ na presença de 1, 3 e 10 g/mL de HCP.

\begin{tabular}{|c|c|c|c|}
\hline AGONISTA & Concentração de HCP & $\left.\mathrm{CE}_{50} \mu \mathrm{g} / \mathrm{mL}\right)$ & Emax\% \\
\hline \multirow{4}{*}{ 蛋 } & $0 \mu \mathrm{g} / \mathrm{mL}$ & $9,85 \times 10^{-7}$ & 100 \\
\hline & $1 \mu \mathrm{g} / \mathrm{mL}$ & $8,51 \times 10^{-7}$ & $102,8 \pm 7,2$ \\
\hline & $3 \mu \mathrm{g} / \mathrm{mL}$ & $1,05 \times 10^{-6}$ & $67,4 \pm 16,3$ \\
\hline & $10 \mu \mathrm{g} / \mathrm{mL}$ & $2,03 \times 10^{-6}$ & $49,2 \pm 12,2 *$ \\
\hline \multirow{4}{*}{ 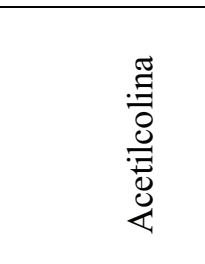 } & $0 \mu \mathrm{g} / \mathrm{mL}$ & $6,76 \times 10^{-8}$ & 100 \\
\hline & $1 \mu \mathrm{g} / \mathrm{mL}$ & $6,74 \times 10^{-8}$ & $93,8 \pm 10,1$ \\
\hline & $3 \mu \mathrm{g} / \mathrm{mL}$ & $5 \times 10^{-8}$ & $88,3 \pm 15,1$ \\
\hline & $10 \mu \mathrm{g} / \mathrm{mL}$ & $5,3 \times 10^{-8}$ & $79,9 \pm 20,1$ \\
\hline \multirow{4}{*}{ 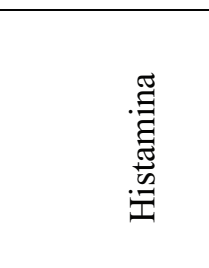 } & $0 \mu \mathrm{g} / \mathrm{mL}$ & $3,93 \times 10^{-8}$ & 100 \\
\hline & $1 \mu \mathrm{g} / \mathrm{mL}$ & $3,85 \times 10^{-8}$ & $98,1 \pm 7,8$ \\
\hline & $3 \mu \mathrm{g} / \mathrm{mL}$ & $5,92 \times 10^{-8}$ & $101,6 \pm 1,6$ \\
\hline & $10 \mu \mathrm{g} / \mathrm{mL}$ & $1,13 \times 10^{-7}$ & $92,9 \pm 8,0$ \\
\hline \multirow{4}{*}{$\vec{v}$} & $0 \mu \mathrm{g} / \mathrm{mL}$ & $1,23 \times 10-3$ & 100 \\
\hline & $1 \mu \mathrm{g} / \mathrm{mL}$ & $1,03 \times 10^{-3}$ & $102,7 \pm 3,4$ \\
\hline & $3 \mu \mathrm{g} / \mathrm{mL}$ & $8 \times 10^{-4}$ & $100,2 \pm 25,6$ \\
\hline & $10 \mu \mathrm{g} / \mathrm{mL}$ & $2,37 \times 10^{-3}$ & $75,7 \pm 7,3$ \\
\hline
\end{tabular}

Os dados representam a média de 5 experimentos \pm EP. * diferença significativa em relação ao controle, ANOVA F ${ }_{3,19}=4,36 \mathrm{p}<$ 0,05 


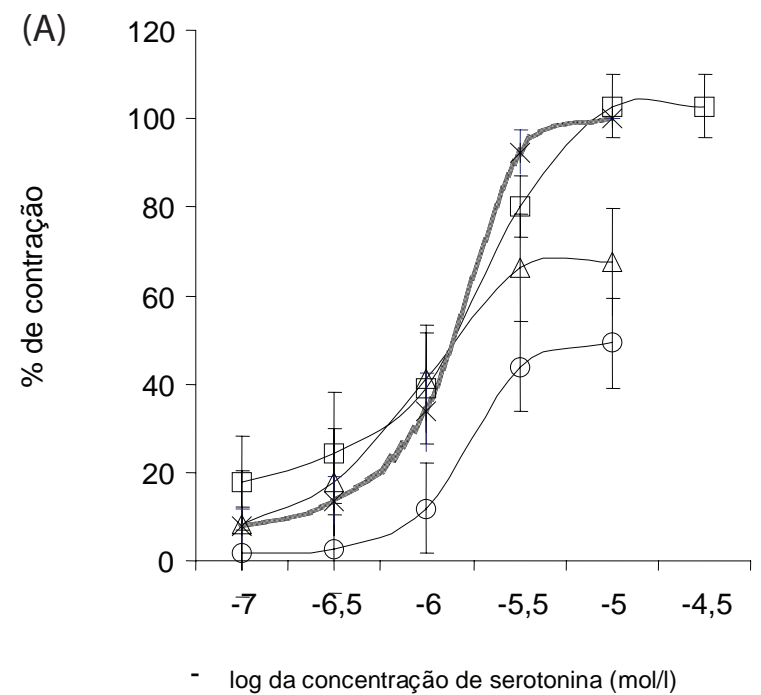

(C)

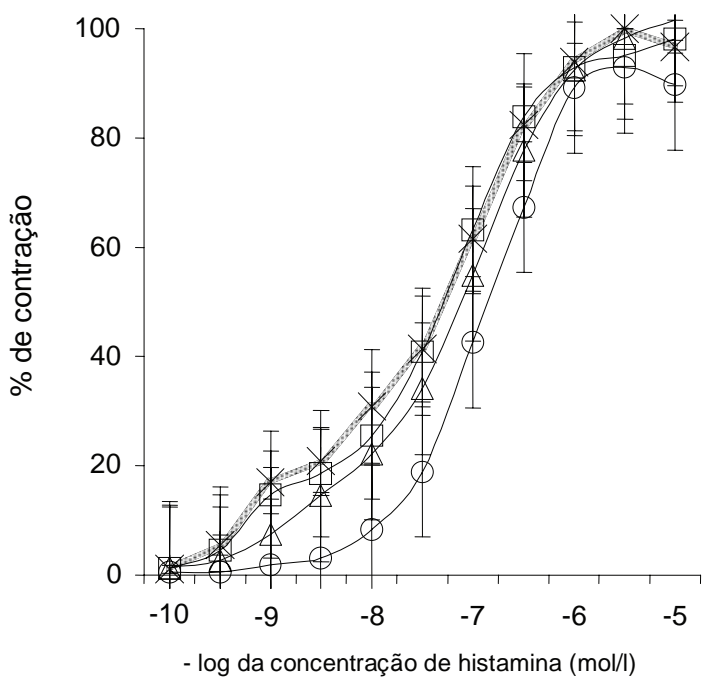

(B)

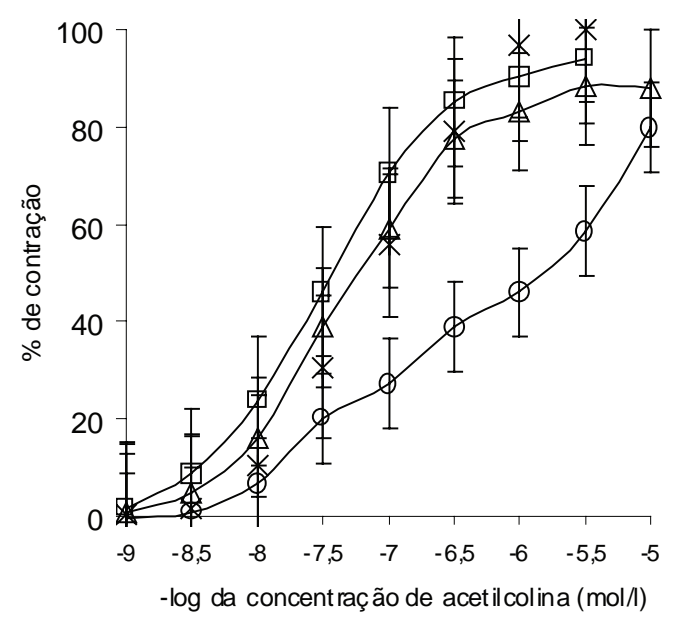

(D)

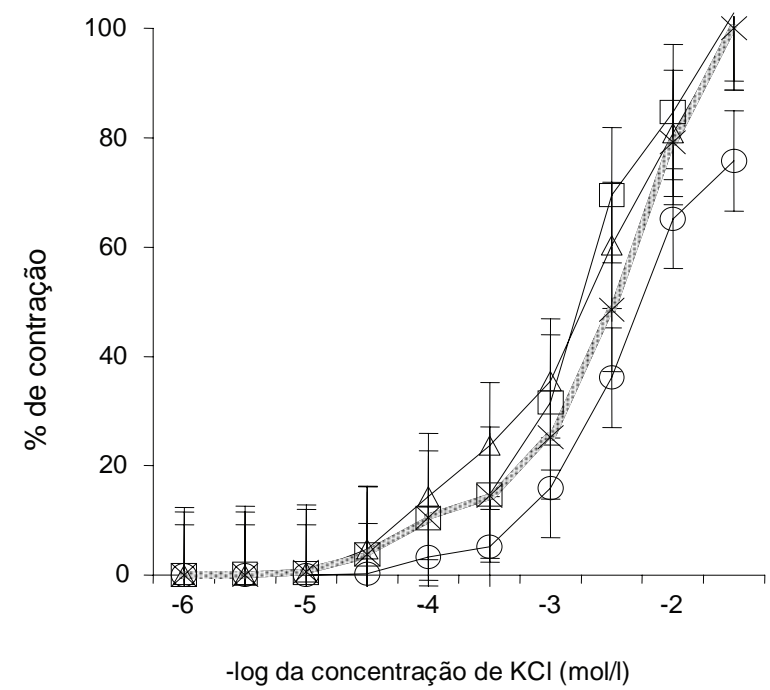

Figura 1. Curvas log concentração-efeito obtidas em íleo isolado de porquinho-da-índia (Tyrode, $37{ }^{\circ} \mathrm{C}$, registro isotônico, $1 \mathrm{~g}$ de carga e 6x de ampliação) com serotonina (A), acetilcolina (B), histamina (C) e cloreto de potássio (D) na presença de concentrações crescentes $(\mu \mathrm{g} / \mathrm{mL})$ de HCP : $-\mathrm{x}-0 ;-\square-1 ;-\Delta-3 ;-\mathrm{O}-10$. Média de 5 experimentos \pm EPM. Redução significativa do efeito máximo (ANOVA $\left.\mathrm{F}_{3,19}=4,36 ; \mathrm{p}<0,05\right)$.

competitivo e/ou não-competitivo foi avaliado por análise de variância (ANOVA) comparando-se o $\mathrm{pD}_{2} \mathrm{e}$ efeito máximo, respectivamente, na presença e ausência de extrato.

\section{RESULTADOS E DISCUSSÃO}

HCP foi testado nas concentrações de 1, 3 e $10 \mathrm{mg} /$ $\mathrm{mL}$ na presença dos agonistas: serotonina, acetilcolina, histamina e cloreto de potássio. A concentração de 30 $\mathrm{mg} / \mathrm{mL}$ foi analisada, mas inibiu qualquer resposta dos agonistas. As concentrações utilizadas não alteraram a tensão basal do íleo. As curvas concentração-efeito são apresentadas na Figura 1.

A Tabela 1 apresenta as variáveis farmacológicas: concentração efetiva $50 \%\left(\mathrm{CE}_{50}\right)$, e efeito máximo (Emax\%).

A análise de variância indicou que a inibição do efeito máximo de ACh, histamina e $\mathrm{KCl}$ (c.a. 20; 7 e 25 $\%$, respectivamente) não foi significativa, demonstrando que o extrato não possui efeito direto sobre os elementos contráteis do órgão. Apenas na presença $10 \mathrm{mg} / \mathrm{mL}$ de HCP foi observada inibição significativa do efeito máximo de serotonina. O valor de pD’2, obtido pelo 


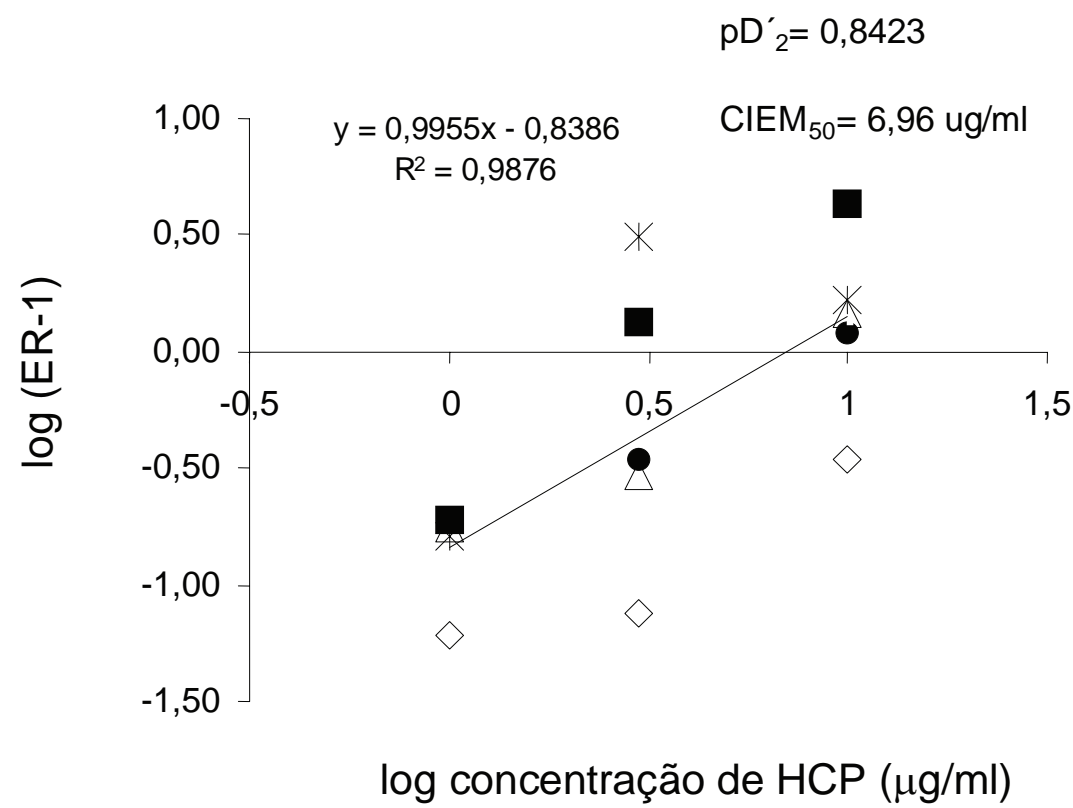

Figura 2. Gráfico de Schild modificado. Relação entre log (ER-1) de serotonina e log da concentração de HCP. Cada ícone representa um experimento e a linha de tendência, a média.

gráfico de Schild modificado (Figura 2) foi de 0,84. O coeficiente de correlação foi $\mathrm{R}^{2}=0,98$ e o coeficiente angular (inclinação da reta) de 0,99 sugeriram a adequação do método para a avaliação. O efeito máximo da serotonina foi reduzido, significativamente, em 50,8 \%. A CIEM50 (concentração inibidora do efeito máximo em $50 \%$ ) da serotonina, calculada a partir do antilogaritmo da concentração de HCP, foi de 6,96 mg/ $\mathrm{mL}$.

Embora algumas espécies nativas sejam utilizadas para problemas gastrintestinais na medicina popular (Corrêa, 1984; Mentz et al., 1997), nenhum estudo direcionado a este tipo de atividade foi encontrado. A espécie européia, $H$. perforatum, inibiu em 98 \% a contração causada por serotonina (Chatterjee et al., 1998), na concentração de $10 \mathrm{mg} / \mathrm{mL}$ do extrato preparado com $\mathrm{CO}_{2}$ supercrítico, rico no floroglucinol hiperforina (38,8 \%). Os autores relatam que a hiperforina inibiu não apenas as contrações induzidas por 5-HT, como também por vários outros agentes espasmogênicos testados, sendo assim um espasmolítico inespecífico. Em nossos experimentos, HCP, que contém cerca de $8 \%$ do floroglucinol HC1 (Viana et al., 2005), apresentou efeito apenas sobre as curvas de serotonina reduzindo o efeito máximo em cerca de $50 \%$, indicativo de antagonismo nãocompetitivo e específico para serotonina. A análise da Figura 1 também revela um deslocamento para direita da curva de acetilcolina na presença de $10 \mathrm{mg} / \mathrm{mL}$ de HCP, entretanto este deslocamento não foi significativo para indicar antagonismo competitivo.
Estes resultados permitem sugerir que a analgesia observada por Viana et al. (2003) com o pré-tratamento com o HCP possa estar relacionada à diminuição da contração peritônio-visceral mediada pela ativação de receptores serotonérgicos. Os subtipos de receptores serotoninérgicos majoritariamente encontrados no intestino são 5- $\mathrm{HT}_{3}$ e 5- $\mathrm{HT}_{4}$ (Tyers; Freedman, 1992, Hegde et al., 1995; Degen et al., 2001; Costall; Naylor, 2004). As substâncias antagonistas $5-\mathrm{HT}_{3}$, inibidoras da motilidade gástrica, são as mais relevantes na clínica, sendo que medicamentos como ondasetrona e granisetrona são utilizados no controle de náusea e vômito causados pela terapia anti-câncer (Costall; Naylor, 2004; Brandão, 2004; Herrstedt et al., 2005). Deste modo, o estudo da atividade de $H$. caprifoliatum sobre receptores periféricos, especialmente serotoninérgicos, pode ser uma perspectiva interessante na busca de moléculas com atividade sobre a motilidade gastrintestinal.

\section{REFERÊNCIAS}

Amaral KM 2000. Estudo de toxidade reprodutiva em ratas Wistar e da atividade antiespasmódica in vitro de Passiflora alata Dryander e Passiflora edulis Sims. Dissertação de Mestrado: Porto Alegre, UFRGS, 84p.

Äriens EJ, van Rossum JM 1957. $\mathrm{pD}_{2}, \mathrm{pA}_{2}$ and $\mathrm{pD}_{2}^{\prime}$ Values in the analisys of pharmacodynamics. Arch Int Pharmacod 110: 275-299.

Bilia AR, Gallori S, Vincieri FF 2002. St. John's wort and depression: Efficacy, safety and tolerability - na update. Life Sci 70: 3077-3096. 
Blumenthal M (Ed.) 1998. The Complete German Commission E Monographs, Therapeutic Guide to Herbal Medicines. Austin: American Botanical Council.

Bjorkman R 1995. Central antinociceptive effects of nonsteroidal anti-inflammatory drugs and paracetamol. Experimental studies in the rat. Acta Anaesth Scand Suppl 103:1-44.

Brandão ABM 2004. Farmacologia clínica do sistema digestivo. In: Fuchs FD, Wannmacher L, Ferreira MBC (ed) Farmacologia Clínica: Fundamentos da terapêutica racional. Rio de Janeiro: Guanabara Koogan 3ed.

Chatterjee SS, Nöldner M, Koch E, Erdelmeier C 1998. Antidepressant activity of Hypericum perforatum and hiperforin: the neglected possibility. Pharmacopsychiatry 31: 7-15.

Corrêa P 1984. Dicionário das plantas úteis do Brasil e das exóticas cultivadas. Rio de Janeiro.

Costall B, Naylor RJ 2004. 5-HT3 receptors. Curr Drug Targets CNS Neurol Disord 3: 27-37.

Dall'agnol R, Ferraz A, Bernardi AP, Albring D, Nör C, Sarmento L, Lamb L, Hass M, von Poser G, Schapoval EES 2003. Antimicrobial activity of some Hypericum species. Phytomedicine 10: 511-516.

Daudt R, von Poser GL, Neves G, Rates SMK 2000. Screening for the anti-depressant activity of some species of Hypericum from South Brazil. Phytother Res 14: 344-346.

Degen L, Matzinger D, Merz M, Appel-Dingemanse S, Osborne S, Lüchinger S, Bertold R, Maecke $\mathrm{H}$, Beglinger C 2001. Tegaserod, a 5-HT4 receptor partial agonist, accelerates gastric emptying and gastrointestinal transit in healthy male subjects. Aliment Pharm Therap 15: 1745-1751.

Duke JA 1985. Handbook of Medicinal Herb. Boca Raton: CRC Presss.

Ernst E 2000. Herbal medicines: where is the evidence? Brit Med J 321: 395-396.

Fenner R, Sortino M, Rates SMK, Dall'agnol R, Ferraz A, Bernardi AP, Albring D, Nör C, von Poser G, Schapoval E, Zacchino S 2005. Antifungal activity of some Brazilian Hypericum species. Phytomedicine 12: 236-240.

Ferraz ABF, Faria DH, Benneti MN, Brondani da Rocha A, Schwartsmann G, Henriques AT, von Poser GL 2005. Screening for antiproliferative activity of six southern Brazilian species of Hypericum. Phytomedicine 12: 112-115.

Fritz D, Bernardi AP, Haas JS, Ascoli BM, Bordignon SAL, von Poser G 2007. Germination and growth inhibitory effects of Hypericum myrianthum and H. polyanthemum extracts on Lactuca sativa L. Rev Bras Farmacogn 17: 44-48.

Gnerre C, von Poser GL, Ferraz A, Viana AF, Testa B, Rates SMK 2001. Monoamine oxidase inhibitory activity of some Hypericum species native to South Brazil. J Pharm Pharmacol 53: 1273-1279.

Hegde SS, Wong AG, Perry MR, Ku P, Moy TM, Loeb M, Eglen R M 1995. 5-HT4 receptor mediated stimulation of gastric emptying in rats. N-S Arch Pharmacol 35: 589-595.

Herrstedt J, Koeller JM, Roila F, Hesketh PJ, Warr D, Rittenberg C, Dicato M 2005. Acute emesis: moderately emetogenic chemotherapy. Support Care Cancer 13: 97-103.

Kasper S, Anghelescu I, Szegedi A, Dienel A, Kieser M 2006. Superior efficacy of St Johns wort extract WS(R) 5570 compared to placebo in patients with major depression: a randomized, double-blind, placebocontrolled, multi-center trial [ISRCTN77277298]. BMC Medicine.

Le Bars D, Gozariu M, Cadden SW 2001. Acute pain measurement in animals. Part 1. Ann Fr Anesth Reanim 20: 347-465.

Linde K, Knuppel L 2005. Large-scale observational studies of hypericum extracts in patients with depressive disorders-a systematic review. Phytomedicine 12: 148-157.

Malamas M, Marselos M 1992. The tradition of medicinal plants in Zagori, Epirus (northwestern Greece). $J$ Ethnopharmacol 37: 197-203.

Mentz LA, Lutzemberger LC, Schenkel EP 1997. Da flora medicinal do Rio Grande do Sul: notas sobre a obra de D’Avila (1910). Cadernos de Farmácia 15: 2547.

Nepomuceno F, Las Casas L, Peters VM, Guerra MO 2005. Desenvolvimento embrionário em ratas tratadas com Hypericum perforatum durante o período de implantação. Rev Bras Farmacogn 15: 224-228.

Reichling J, Weseler A, Saller RA 2001. Current review of the antimicrobial activity of Hypericum perforatum L. Pharmacopsychiatry 34: 116-118.

Reynolds J (Ed) 1993. Martindale The Extra Pharmacopoeia. $30^{\text {th }}$ ed. London: Pharmaceutical Presss.

Rocha L, Marston A, Kaplan MAC, Stoeckli-Evans H, Thull U, Testa B, Hostettmann K 1994. An antifungal gammapyrone and xanthones with monoamine oxidase inhibitory activity from Hypericum brasiliense. Phytochemistry 36: 1381-1385.

Rocha L, Marston A, Potterat O, Kaplan MAC, StoeckliEvans H, Hostettmann K 1995. Antibacterial phloroglucinols and flavonoids from Hypericum brasiliense. Phytochemistry 40: 1447-1452.

Schmitt AC, Ravazzolo AP, von Poser GL 2001. Investigation of some Hypericum species native to Southern of Brazil for antiviral activity. J Ethnopharmacol 77: 239-245.

Tyers MB, Freeman AJ 1992. Mechanism of the anti-emetic activity of 5-HT3 receptor antagonists. Oncology 49: 263-268

Viana AF, Heckler AP, Fenner R, Rates SMK 2003. Antinociceptive activity of Hypericum caprifoliatum and Hypericum polyanthemum (Guttiferae). Braz J Med Biol Res 36: 631-634.

Viana AF, Rego J-C, von Poser G, Ferraz A, Heckler AP, Costentin J, Rates SMK 2005. The antidepressant like effect of Hypericum caprifoliatum Cham \& Schlecht (Guttiferae) on forced swimming test results from an inhibition of neuronal monoamine uptake. Neuropharmacology 49: 1042-1052. 